\title{
The use of abatacept in debilitating cavitating lung disease associated with rheumatoid arthritis, bronchocentric granulomatosis and aspergillosis
}

\author{
K Neff, ${ }^{1}$ J Stack, ${ }^{2}$ S Harney, ${ }^{2}$ M Henry ${ }^{1}$
}

${ }^{1}$ Department of Respiratory Medicine, Cork University Hospital, Cork, Ireland ${ }^{2}$ Department of Rheumatology, Cork University Hospital, Cork, Ireland

\section{Correspondence to}

Karl Neff, 30 Eccles St Dublin 7, Ireland;

karljneff@gmail.com

Received 29 October 2009 Accepted 30 January 2010

\begin{abstract}
A case of debilitating cavitating lung disease associated with rheumatoid arthritis and bronchocentric granulomatosis, which failed to respond to conventional medical or surgical treatment, is described. The patient was treated over 10 years with steroids, antimicrobial agents, disease-modifying antirheumatoid drugs and surgery. Lung function continued to decline and the patient presented for admission with recurrent pneumonia. Abatacept was initiated to modify the underlying immunopathology. Following 12 months of treatment with abatacept the patient has demonstrable improvement in lung function and lung anatomy, and has not presented to hospital with pneumonia. She has tolerated the treatment without complication. The use of abatacept has stabilised the lung disease in this case in the medium term and prevented readmission to hospital. These results suggest a larger role for abatacept in those with such disease in the future and may warrant further investigation.
\end{abstract}

Bronchocentric granulomatosis has been described in association with rheumatoid arthritis (RA) and allergic bronchopulmonary aspergillosis (ABPA). Here we describe the effective use of abatacept in the case of a woman diagnosed with bronchocentric granulomatosis in association with RA, and subsequently in the setting of colonisation by Aspergillus fumigatus. She had been suffering with recurrent pulmonary sepsis, and the use of abatacept has been effective in stabilising her disease.

A 43-year-old woman presented to hospital in 1998 with a suppurative cavitating right upper lobe pneumonia. The patient had a background history of RA that had been diagnosed in 1992 when the patient was 37 years old and was at that time well controlled with hydroxychloroquine. She had no background history of asthma or other respiratory disease at that time. The pneumonia proved resistant to conventional treatment and required right upper lobectomy. Histological analysis of the resected specimen reported bronchocentric granulomatosis and foci of organising pneumonia with multinucleate giant cells and pallisading histiocytes lining the cavity of the resected lobe. Eosinophilia was not conspicuous at that time, and direct staining and culture for tuberculosis (TB) and fungi were negative.

She represented in 2004 with recurrent lower respiratory tract infections. Serial CT imaging of the thorax showed enlarging bilateral apical cavities with mediastinal lymphadenopathy, tree-in-bud opacification and localised bronchiectasis in the right upper lobe. This bronchiectasis was not central in location. Serum antinuclear cytoplasmic antibody (ANCA) was negative. Bronchoscopy did not reveal any endobronchial lesion; however, bronchial washings were positive for A fumigatus. Serum immunoglobulin E (IgE) levels were markedly elevated at $1263 \mathrm{kU} / \mathrm{l}$, RAST (radioallergosorbent test) IgE was $4(0-6)$ and the patient was noted to have an eosinophilia of $1.74 \times 10^{9} / 1$.

During this period the patient's RA flared intermittently, requiring treatment with diseasemodifying antirheumatic agents (DMARDs). Hydroxychloroquine was switched to methotrexate in 2004 but discontinued shortly afterwards due to patient intolerance. Lefluonomide was then introduced but had to be discontinued in view of the recurrent infective episodes. The patient's RA was then managed with reducing courses of steroids which were tapered to a maintenance dose of $10 \mathrm{mg}$ daily.

Again, intensive treatment with broad-spectrum antibiotics and antifungal therapy proved unsuccessful, resulting in recurrent hospital admissions over the following 18 month period. Subsequently, in October 2006, the patient underwent left upper lobectomy. Examination of the resected specimen found bronchocentric granulomatosis with A fumigatus identified within the cavity. TB staining and cultures were negative.

Repeated courses of antimicrobial agents, including itraconazole and voriconazole, were prescribed postresection but were discontinued following a 6 month treatment period with no clinical response. The antifungal agents were prescribed with high dose steroids (maximum dose $=40 \mathrm{mg}$ ), which were continued for 6 weeks postresection and then tapered again to a maintenance dose of $10 \mathrm{mg}$. The patient continued to present with recurrent respiratory tract infections and progressive cavitating lung disease. Repeated bronchoscopies at this time did not isolate any TB or fungal pathogens.

During the postoperative period the patient's nutritional status and weight remained stable. The patient's RA did flare again, and low dose lefluonamide (10 mg daily) was cautiously reintroduced in February 2008 with minimal response. The patient's RA remained active, with a swollen joint count of 10 and a C-reactive protein (CRP) of $252 \mathrm{mmol} / \mathrm{l}$ noted immediately prior to starting abatacept. This CRP was taken in the absence of concurrent respiratory sepsis.

A trial of abatacept was commenced at 20 months postlobectomy in an effort to control her underlying disease given the lack of response to conventional treatment. A dosage of $750 \mathrm{mg}$ was 
administered at monthly intervals and, to date, the patient has completed 12 infusions of abatacept over 12 months.

Our patient has responded well to abatacept and, since commencement of treatment, she has not required further admission to hospital. An improvement is also seen in her pulmonary function tests, with tests prior to and after 12 months of treatment recording an improvement in forced vital capacity (FVC) from 2.41 (71\% predicted) to 2.87 (95\% predicted) and an improvement in forced expiratory volume in $1 \mathrm{~s}\left(\mathrm{FEV}_{1}\right)$ of 1.89 (68\% predicted) to 2.28 (88\% predicted). Lung anatomy has also improved on CT imaging. Figures 1 and 2 illustrate this improvement. Clinically, the patient's arthritis is quiescent and CRP measurements have fallen from $252 \mathrm{mmol} / 1$ on commencement of abatacept to $<5 \mathrm{mmol} / \mathrm{l}$ at 12 months of treatment.

\section{DISCUSSION}

Bronchocentric granulomatosis is a histological diagnosis that is associated with numerous diseases including RA and ABPA. Bronchocentric granulomatosis was identified in our patient on two separate occasions; first in the setting of RA and secondly in the setting of both RA and Aspergillus colonisation. There were therefore two aetiologies: a hypersensitivity reaction and an autoimmune process, which were then potentially accelerated by exposure to bacterial antigens.

Previously, treatment of bronchocentric granulomatosis-associated lung disease has focused on treating the underlying condition. In cases without an obvious cause, steroids have been used with some success. ${ }^{1}$ There are no previous reports in the literature regarding the use of abatacept in bronchocentric granulomatosis, although there have been cases of omalizumab in treating ABPA in the context of cystic fibrosis, with impressive results. ${ }^{2}$

Our patient had severe cavitating lung disease that was resistant to standard medical treatment with antimicrobial agents, immunosuppressant agents and surgery. The underlying mechanism is probably multifactorial, but proteolytic enzyme activity is likely to be an important part of the condition given the concomitant RA and the anatomical appearance. In this case, the proteolysis could be stimulated by proinflammatory pathways, which can be activated or catalysed by tumour necrosis factor $\alpha(\mathrm{TNF} \alpha)^{4}$

Anti-TNF treatment was considered for the patient's systemic arthritis, but case reports have shown infliximab (chimeric monoclonal antibody to TNF) to have worsened $\mathrm{ABPA}^{5}$ and thus these particular agents were not used in this case.

In RA there are several co-stimulatory and co-inhibitory pathways, and signals through these can either upregulate or

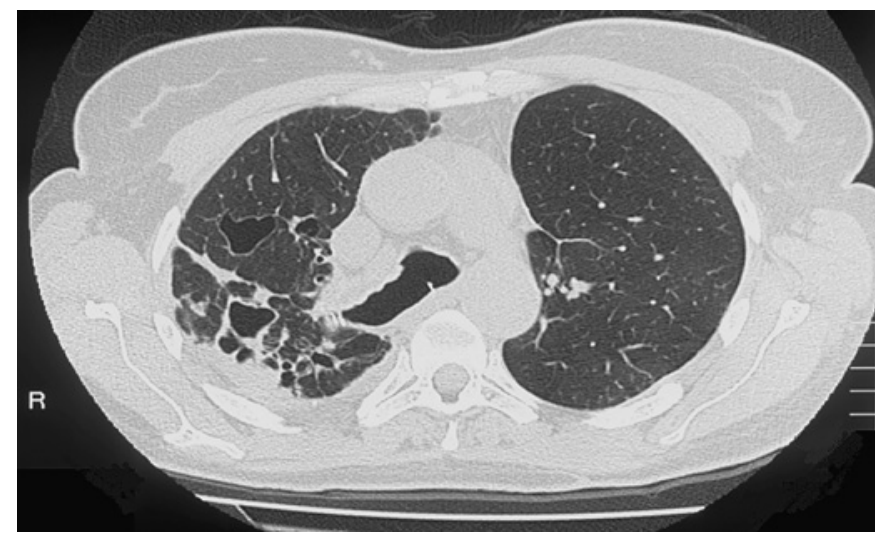

Figure 1 CT before abatacept treatment. Cavities are illustrated in the right middle and lower lobes.

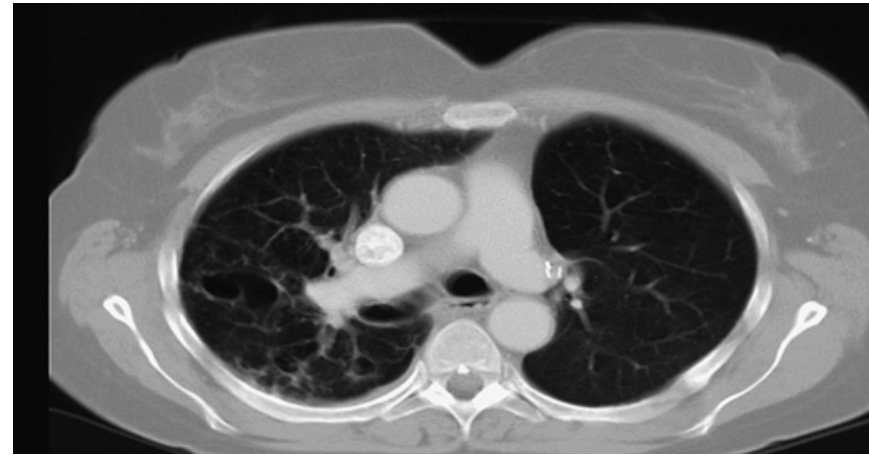

Figure 2 CT thorax 12 months after onset of abatacept. An improvement in appearance of cavities in the right middle and lower lobes can be seen.

downregulate $\mathrm{T}$ cell activation, an important process in RA pathogenesis. Upregulation of $\mathrm{T}$ cell activity via the activation of co-stimulatory pathways may have facilitated the immune response to Aspergillus, where chemotaxis of $\mathrm{CD}^{+}{ }^{+} \mathrm{T}$ helper 2 (Th2) lymphocytes specific for A fumigatus has been shown to occur. ${ }^{6}$ Abatacept (which inhibits T cell co-stimulation) was therefore chosen as a preferable biological agent for treatment of both the patient's RA and lung disease. Rates of TB are certainly lower with abatacept than with anti-TNF agents and also overall infection rates are lower with abatacept. ${ }^{7}$

Abatacept is a soluble fusion protein between CTLA-4 (cytotoxic T-lymphocyte-associated antigen 4) and the Fc portion of IgG1 (CTLA4-Ig).

In our case, the use of abatacept proved successful in treating both the patient's bronchocentric granulomatosis-associated cavitating lung disease and her arthritis. We propose that abatacept may also have played a role in suppressing the underlying Aspergillus-associated bronchocentric granulomatosis in our patient via $\mathrm{T}$ cell inhibition.

\section{CONCLUSION}

Treatment with abatacept in this case has resulted in a significant improvement in the patient's clinical condition from both a respiratory and an arthritis point of view. This has led to a reduced incidence of infection, prevented hospital admission and enhanced our patient's quality of life. As such, we believe this to be the first case report of abatacept being used successfully in the treatment of bronchocentric granulomatosis-associated lung disease with co-commitant RA.

\section{Competing interests None.}

Patient consent Obtained.

Provenance and peer review Not commissioned; externally peer reviewed.

\section{REFERENCES}

1. Westhoff M, Welim B, Müller KM. Bronchocentric granulomatosis (in German). Pneumologie 2005;59:804-10.

2. Van der Ent CK, Hoekstra H, Rijkers GT. Successful treatment of allergic bronchopulmonary aspergillosis with recombinant anti-lgE antibody. Thorax 2007:62:276-7.

3. Kanu A, Patel K. Treatment of allergic bronchopulmonary aspergillosis (ABPA) in CF with anti-lgE antibody (omalizumab). Pediatr Pulmonol 2008;43:1249-51.

4. Moreland LW, Baumgartner SW, Schiff MH, et al. Treatment of rheumatoid arthritis with a recombinant human tumor necrosis factor receptor (p75)-Fc fusion protein. N Engl J Med 1997;337:141-7.

5. Judson MA. Allergic bronchopulmonary aspergillosis after infliximab therapy for sarcoidosis: a potential mechanism related to T-helper cytokine balance. Chest 2009;135:1358-9.

6. Knutsen AP, Chauhan B, Slavin RG. Cell-mediated immunity in allergic bronchopulmonary aspergillosis. Immunol Allergy Clin N Am 1998;18:575-99.

7. Salliot C, Dougados M, Gossec L. Risk of serious infections during rituximab, abatacept and anakinra treatments for rheumatoid arthritis: meta-analyses of randomised placebo-controlled trials. Ann Rheum Dis 2009;68:25-32. 